Rev. Elev. Méd. vét. Pays trop., 1971, 24 (4) : 551-63

\title{
Les glossines (Diptera, glossinidae) du Sénégal : Ecologie, répartition géographique et incidence sur les trypanosomoses
}

\author{
par S. M. TOURE (*)
}

\begin{abstract}
RESUME
Cette note présente une synthèse des prospections faites entre 1965 et 1968 pour préciser la répartition géographique et l'écologie des glossines au Sénégal. Trois espèces de glossines sont mentionnées: Glossina morsitans submorsitans Newstead, 1910; G. longipalpis Wiedemann, 1830; G. palpalis gambiensis Vanderplank, 1949.

Les glossines occupent le sud du Sénégal, à l'exception toutefois de quelques îlots de G. palpalis gambiensis, situés le long de la côte atlantique dans des palmeraies à huile ou des marigots à végétation dense.

Dans la mangrove de l'embouchure du Saloum $G$. palpalis gambiensis est fréquente, cependant que $G$. morsitans se trouve dans les forêts claires.

La plus grande fréquence des glossines a été constatée en Casamance et au Sénégal oriental. Dans ces régions, G. palpalis gambiensis se trouve dans plusieurs types de formations botaniques: les forêts denses humides, les palmeraies, la mangrove et la végétation riveraine des cours d'cau. G. morsitans submorsitans a une vaste distribution dans les forêts claires et la savane arborée. $G$ longipalpis, mentionnée par des travaux antérieurs, n’a pas été décelée au cours de ces prospections.

Dans l'aire occupée par les glossines, les animaux domestiques hébergent une ou plusieurs espèces de trypanosomes: Trypanosoma brucei, $T$. congolense, $T$. virux, mais la trypanosomose est relativement bien tolérée en Casamance et dans la Haute Gambie (où le bétail est de race Ndama) par rapport à d'autres régions à élevage de zébus.

Cette étude est illustrée par une carte générale de répartition des glossines.
\end{abstract}

\section{INTRODUCTION}

Les premières mentions de localisation géographique des espèces de glossines rencontrées au Sénégal remontent au début du siècle et sont dues à des médecins, épidémiologistes de la maladie du sommeil (A. THIROUX, 1909; A. THIROUX et L. d'ANFREVILLE DE LA SALLE, 1911). Elles sont suivies quelques années plus tard par les prospections entomo-

(*) Institut d'Elevage et de Médecine vétérinaire des Pays tropicaux; Laboratoire national de l'Elevage et de Recherches vétérinaires, Dakar-Hann, B.P. 2057. logiques d'E. ROUBAUD faites sur la PetiteCôte du Sénégal et dans le Bas-Saloum. En 1912, M.W. DUFOUGERE publie une étude sur la maladie du sommeil et les trypanosomoses animales en Casamance. BOUET parcourt, en 1916, les zones à glossines du Sénégal en longeant le chemin de fer transcontinental de Thiès à Kayes. Faisant le bilan de leurs travaux sur la lutte contre la maladie du sommeil sur la Petite-Côte et dans les Niayes du Sénégal, M. MOULANIER et J. DIOUF, 1952, mentionnent les villages les plus atteints dans ces régions, en rapport avec la présence de glossines. L'étude de la réparti- 
tion géographique de celles-ci est reprise par P.C. MOREL et S.M. TOURE, 1964-1967. Citons enfin lcs récentes enquêtes écologiques et épidémiologiques de A. CHALLIER, 1970, menées sur la Petite-Côte, dans le foyer de trypanosomose de la Somone.

A ces prospections, faites au Sénégal, s'ajoutent plusieurs cartes d'épidémiologie des trypanosomoses ou de répartition géographique des glossines dans l'Ouest africain : P. GOUZIEN, 1908; E. ROUBAUD, 1922; H. GASCHEN, 1945; les Services géographiques de l'A.O.F., 1949; P. MORNET, 1953; W.H. POTTS, 1953; A. RICKENBACH, 1961.

La présente note indique les résultats des enquêtes menées de 1965 à 1968 pour définir avec plus de précision la répartition géographique des glossines au Sénégal et la fréquence relative des trypanosomoses du bétail. Elle fait suite à une première note (S.M. TOURE, 1968) qui résumait ces résultats.

\section{METHODES}

Pour des raisons d'unité topographique, les prospections ont été menées par région administrative en utilisant la carte du Sénégal au 1/200,000. Ces prospections, objet de plusieurs rapports, ont conduit à des cartes de répartition où les lieux de capture des glossines sont marqués d'un signe conventionnel. Chacune de ces cartes, à l'exclusion du Sénégal oriental considère une portion du territoire évaluée à $1.200 \mathrm{~km}^{2}$ environ, correspondant à un carré dont les côtés sont de 20 minutes en latitude comme en longitude, soit environ $35 \mathrm{~km}$. Pour le Sénégal oriental, en raison des difficultés de circuler dans les réserves forestières et du fait de la rareté des pistes, les prospections ont été faites à l'aide de cartes au 1/500.000. La carte finale de distribution est la synthèse au millionième de toutes les précédentes cartes.

\section{ESPECES PRESENTES ET FREQUENCE RELATIVE}

- Sous-genre Glossina Wiedemann sensu stricto :

Glossina morsitans submorsitans Newstead, 1910;
Glossina longipalpis Wiedemann, 1830.

- Sous-genre Nemorhina Robineau - Desvoidy :

Glossina palpalis gambiensis Vanderplank, 1949.

Glossina palpalis gambiensis est rencontrée dans des plages de végétation particulière le long de la Grande-Côte et de la Petite-Côte atlantiques: Niayes, Nougouna, Somone. Plus au sud, l'espèce est abondante dans l'embouchure du Saloum et en Casamance.

Glossina morsitans submorsitans cst, de loin, l'espèce la plus fréquente en nombre et en étendue.

Glossina longipalpis, mentionnée plusieurs fois en Casamance, n'a cependant pas été décelée dans les aires que lui avait assignées W. H. POT'TS en 1953. L'espèce ne figure pas dans les collections que nous avons pu consulter : collections établies par P.C. MOREL depuis 1956 au Laboratoire national de l'Elevage et de Recherches vétérinaires, Dakar; collections de l'Institut fondamental d'Afrique noire. Cette constatation ne permet cependant pas d'infirmer son existence et nous reprenons pour mémoire les aires probables de répartition selon POTTS, tout en considérant l'espèce comme très rare.

\section{REPARTITION GEOGRAPHIQUE ET ECOLOGIE}

\section{Les îlots résiduels de Glossina palpalis}

Quelques gites à Glossina palpalis gambiensis, qui devaient primitivement se rattacher à de grandes aires infestées, persistent le long des côtes atlantiques. Il s'agit :

- des Niayes;

- de la Nougouna;

- de la Somone.

Ces îlots sont liés à la présence de marigots alimentés par les pluies d'hivernage (isohyète 500 ).

Dans les Niayes du Sénégal, le biotope des glossines est constitué par des galeries de palmiers à huile (Elaeis guineensis) qui poussent autour de ces marigots. Du point de vue écologique, les glossines trouvent des conditions 
très favorables, assez comparables à celles qui prévalent en zone subguinéenne plus au sud.

La végétation du lit permanent de la Nougouna est composée d'un grand nombre de plantes ripicoles, notamment des Ficus à racines-échasses. Cela favorise le maintien d'une humidité relative élevée, propice au développemcnt des glossines dans la végétation périphérique du marigot.

Quant à la Somone, son cours principal s'étend sur 12 à $13 \mathrm{~km}$, mais elle comporte de nombreux ruisseaux qui l'alimentent en saison des pluies et sont à sec ensuite. La partie côtière est bordée de palétuviers (Avicennia) ou jonchée de Typha, tandis que le cours supérieur se trouve dans une forêt à Acacia et Adansonia.

\section{Les glossines du Sine-Saloum}

Nous trouvons ici deux principaux types d'habitat: à l'ouest les bôlons (marigots salés à l'embouchure du Saloum) et les forêts claires plus à l'est.

Les rives des bôlons sont couvertes de palétuviers où l'on rencontre des glossines. Les palétuviers sont de faible hauteur et reçoivent un rayonnement solaire important; de plus, ils poussent dans une mare impropre à l'oviposition des tsé-tsé. Le présence de glossines dans un tel biotope doit être interprétée comme une conséquence de l'activité de chasse, l'habitat réel de la glossine étant constitué par les formations forestières non édaphiques avoisinant les bôlons. Plusieurs captures de $G$. palpalis gambiensis ont été faites dans ces bôlons entre Foundiougne, Sokone et Sangako.

Les forêts situées à l'est sont des forêts claires de type nord-soudanien (forêts de Vélor, Kousmar et Keur Makhtar). Elles renferment toutes G. morsitans submorsitans en abondance.

\section{La Casamance et le Sénégal oriental}

\section{a) La Basse-Casamance}

Par son réseau hydrographique, sa végétation et le régime des pluies (isohyètes 1.000 $1.250 \mathrm{~mm}$ ), la Casamance occidentale est le prolongement des mosaïques de forêts-savanes de Guinée. La densité des formations forestières et le nombre des cours d'eau ont une incidence marquée sur la répartition des espèces de glossines qui se traduit par une grande fréquence de $G$. palpalis gambiensis. Les principales formations qui abritent cette glossine en Basse Casamance peuvent être classées comme suit :

\section{- Les forêts denses humides}

Elles sont caractérisées par « des peuplements pluristrates, la strate supérieure étant composée de grands arbres à futaie semper virens ". De telles forêts couvrent environ un tiers des étendues prospectées dans cette province. Parmi les arbres qui les constituent, on note le plus souvent le cailcedrat (Khaya senegalensis) : forêts de Koular, de Tobor, de Tindouk; des associations de cailcédrat et de Detarium : forêt des Narangs. Quelques forêts à étendue limitée sont constituées de tecks (Tectona grandis); elles sont assez denses pour être rattachées aux formations denses humides malgré leur caractère artificiel apparent (forêt de Djibélor, forêt des Bayottes). Quelques autres forêts sont composées d'essences diverses sans prédominance particulière de l'une d'elles (forêts de Sambouladian à Ceiba, Khaya, Detarium et Elaeis).

Dans toutes ces formations, G. palpalis gambiensis a été capturée en abondance, souvent à l'exclusion de G. morsitans.

\section{- Les palmeraies}

Les palmeraies à Elaeis guineensis sont surtout localisées autour des bassins d'inondation des rivières et des marigots, transformés en marécages lors des crues de saison des pluies. Il est à noter que la plupart des villages sont à proximité de ces formations pour l'exploitation des palmiers et l'aménagement en rizières des cuvettes inondées.

Ces palmeraies renferment presque toujours G. palpalis gambiensis.

\section{- La mangrove}

Composée de Rhizophora et Avicennia, la mangrove est ici une végétation d'embouchure qui suit les contours des marigots se jetant dans la mer (bólons). Elle trouve sa plus grande extension au sud de Diouloulou (Diana Bôlon, Diouloulou Bôlon, Koular Etoupaye). Les intersections des cours d'eau conduisent à la formation d'îlots recouverts de palétuviers.

G. palpalis gambiensis fréquente les palétuviers mais ne doit pas s'y reproduire car 
cette végétation pousse dans l'eau. La mangrove entretient une humidité relative très élevée, favorable au développement de la glossine en arrière-pays.

\section{- Les forêts claires et la savane boisée}

Un grand nombre de forêts claires devaient être primitivement des forêts denses, dégradées par la suite (forêts de Tabir, Silinnki, Tanguite, Suèle, etc.). Ces formations renferment encore $G$. palpalis mais $G$. morsitans y est plus fréquente. Les savanes situées au nord de la Casamance, le long de la frontière gambienne, renferment $G$. morsitans submorsitans.

La résultante des facteurs botaniques cidessus se traduit en Basse Casamance par une distribution géographique de $G$. palpalis gambiensis en plages étendues, sans discontinuité appréciable, tandis que $G$. morsitans submorsitans occupe des aires nettement plus restreintes, de Ziguinchor à Kolda.

b) La Haute Casamance et le Sénégal oriental

En allant d'ouest en est, les forêts denses humides de la Basse Casamance voicnt leur étendue se restreindre; elles sont supplantées par la forêt claire et la savane. La fréquence relative des espèces de glossines et leur répartition change aussi. On peut distinguer ici trois types d'habitat des glossines:

- Les îlots de palmiers à huile

Localisés dans des dépressions marécageuses à proximité des cours d'eau, ils représentent autant de gîtes à Glossina palpalis gambiensis.

- La végétation riveraine de la Casamance et de la Gambie

On trouve encore, le long des rives, des palétuviers en formation souvent très dense. Là où les cours d'eau ont une faible largeur, la végétation riveraine est souvent constituée d'arbres à racines-échasses (Ficus sp., Pterocarpus sp.). $G$. palpalis gambiensis occupe la plupart de ces formations. Sa distribution géographique est ici linéaire, étroitement liée à la présence d'un cours d'eau.

- La forêt claire et les savanes arborées

La forêt claire est de type relativement humide du fait de la présence de marigots, surtout entre Vélingara et la rivière Koulountou. Elle est composée essentiellement de vène (Pterocarpus orinaceus), dimb (Cordyla africana), kapokier (Bombax costatum), santan
(Daniellia oliveri), ronier [Borassus (flabellifer) aethiopum]. Le sous-bois est composé de Combretaceae en grand nombre.

Glossina morsitans submorsitans occupe pratiquement l'ensemble des forêts claires de Haute Casamance et de Haute Gambie.

Dans la partie la plus orientale du Sénégal, le faciès dominant est aussi la forêt claire, mais les savanes arborées sont plus nombreuses. Les cours d'eau se font plus rares et moins larges et la plupart sont périodiques. Quelquesuns de ces cours d'eau coulent au fond de gorges encaissées, dénudécs ou rcvêtues d'une végétation xérophytique associée à des roniers (Borassus). Il en est ainsi de la Koulountou et du tronçon de la Gambie située à l'ouest de la réserve du Niokolo-Koba.

Dans ces formations, G. morsitans submorsitans est la seule espèce rencontrée.

Ces données écologiques et la juxtaposition des points de capture des glossines (planche $\mathrm{n}^{0} 2$ ) ont conduit à une synthèse cartographique au millionième de la répartition des glossines, exprimée en fréquence relative des espèces.

La plus grande difficulté est de définir avec précision la limite nord de répartition des glossines. Nos prospections permettent de faire remonter la limite nord de distribution au Sénégal oriental jusqu'à $14^{\circ} 40^{\prime}$ latitude nord (Médina Samba Gouro), à une trentaine de kilomètres de Bakel, où $G$. morsitans submorsitans a été effectivement capturée. Au nord de cette localité, il y a un changement notable du faciès botanique qui permet de conclure sinon à l'absence de glossines, du moins à quelques incursions périodiques et transitoires de celles-ci en saison des pluies. L'infection par Trypanosoma congolense de quelques bovins consultés à Bakel est en faveur de cette idée, encore que l'on ne saurait exclure l'éventualité d'une infection contractée dans les forêts à glossines au cours des déplacements du bétail.

Si la limite nord de distribution des glossines se situe à l'est, nettement au-dessus du quatorzième parallèle, il n'est est pas de même dans les forêts limitrophes de Tambacounda. En longeant plusieurs pistes de direction sud-nord, le nombre des captures de glossines a très vite diminué. Cela est lié à une modification du faciès botanique. Le facteur limitant de l'exten- 
sion géographique des glossines est la sécheresse: nous sommes au voisinage du Ferlo, subdésertique et rigoureux. Ces faits ne permettent pas de faire remonter les glossines à plus de $14^{\prime \prime}$ au nord-ouest de Tambacounda.

\section{INCIDENCE SUR LES TRYPANOSOMOSES}

G. palpalis gambiensis transmet au Sénégal :

- Trypanosoma (Trypanozoon) gambiense Dutton, 1902;

- Trypanosoma (Trypanozoon) brucei Plimmer et Bradford, 1899;

- Trypanosoma (Nannomonas) congolense, Broden, 1904;

- Trypanosoma (Dutonella) vivax Ziemann, 1905.

La maladic du sommeil à $T$. gambiense existe en foyers localisés, notamment autour des Niayes et sur la Petite-Côte (foyer de la Somone). Quelques cas sont signalés annuellement en d'autres secteurs d'endémie dans l'aire de G. palpalis gambiensis. Les trypanosomoses du bétail sont observées partout où se trouve cette glossine, avec une plus grande fréquence de $T$. vivax par rapport à $T$. brucei et $T$. congolense.

G. morsitans submorsitans transmet les mêmes espèces de trypanosomes du bétail. à l'exclusion de $T$. gambiense de l'Homme.

Dans l'épizootiologie des trypanosomoses, il y a lieu de distinguer la situation qui prévaut en Casamance et en Haute Gambie, où le bétail est de race $\mathrm{Ndama}$ et tolère la présence des glossines, de celle des autres régions à élevages de zébus ou de croisement ZébuNdama. Dans ces dernières régions, les trypanosomoses posent un problème grave, d'autant plus difficile à résoudre que l'alimentation des animaux est des plus précaires.

\section{CONCLUSION}

La répartition géographique actuelle des glossines au Sénégal n'est pas en tous points conforme à celle qui était admise il y a quelque trente années.

Glossina palpalis gambiensis était signalée le long de la côte occidentale depuis SaintLouis et le Delta du Fleuve Sénégal jusqu'à la frontière de la Gambie. L'espèce s'arrête actuellement au $15^{\circ}$ parallèle. Sa disparition plus au nord est due au déboisement pour les besoins agricoles. Sur la Petite-Côte atlantique, l'espèce n'occupe plus que la vallée de la Nougouna et la Somone. Son aire de grande extension reste la Basse Casamance et les rives des fleuves Casamance et Gambie.

G. morsitans submorsitans a, dans le Sénégal oriental, une plus vaste répartition qu'il n'était admis. On la trouve en suivant la piste de Goudiry à Bakel, jusqu'à une trentaine de kilomètres avant cette dernière ville.

$G$. longipalpis qui était mentionnée en beaucoup d'endroits (même dans les Niayes du Sénégal, ce qui, de notre point de vue, procède d'une erreur de détermination), n'a pas été décelée au cours de nos prospections.

Dans toutes les régions infestées de glossines, les animaux domestiques contractent la trypanosomose due à une ou plusieurs espèces de trypanosomes : $T$. vivax, $T$. congolense, $T$. bru$c e i$, mais la gravité de la maladie dépend pour beaucoup des races bovines élevées et de leur alimentation.

\section{REMERCIEMENTS}

Nous tenons à remercier tous ceux, bien nombreux, qui ont eu l'amabilité de nous apporter leur aide très efficace au cours de nos prospections. Nos remerciements vont en partıculier aux Inspecteurs régionaux et aux agents du Service de l'Elevage ainsi qu'aux agents du Service des Eaux et Forêts qui nous ont toujours donné des informations intéressantes et souvent accompagnés dans nos prospections. 
Tableau récapitulatif des prospections sur les glossines

\begin{tabular}{|c|c|c|c|c|c|}
\hline Localitês & $\begin{array}{l}\text { G. morsitans } \\
\text { submorsitans }\end{array}$ & $\begin{array}{l}\text { G. paipalis } \\
\text { gambiensis }\end{array}$ & Localités & $\begin{array}{l}\text { G. morsitans } \\
\text { submorsitans }\end{array}$ & $\begin{array}{l}\text { G. palpatis } \\
\text { gambiensis }\end{array}$ \\
\hline NIAYES & & + & Kagnarou & & + \\
\hline NOUGOUNA & & + & Sinndian & & + \\
\hline SOMONE & & + & Kalounaye & + & + \\
\hline SOKONE & + & + & Koulayes & & + \\
\hline VELOR & + & & Tapilane & & + \\
\hline DJILOR & + & & Tobor & & + \\
\hline KOUSMAR & + & & Bagaga & & + \\
\hline KEUR MAKHTAR & + & & Diagoune & + & + \\
\hline NGAYENE & + & & Suèle & + & + \\
\hline DIAMA TIEVI & + & & BAILA & & + \\
\hline ZIGUINCHOR & + & + & Tiobon & & + \\
\hline Bayottes & & + & Guirio & & + \\
\hline Banbadinnka & & + & Balonguine & & + \\
\hline Bofa & & + & Kakarē & & + \\
\hline Niadio & & + & Badiana & & + \\
\hline Djibéior & & + & Tindouk & & + \\
\hline Bissine & + & + & Balingor & & + \\
\hline Mpak & & + & Diégoune & & + \\
\hline Oussouye & + & + & SEDHIOU & + & + \\
\hline Kaguite & + & & Boudié & + & + \\
\hline Etafoune & & + & Manëkounda & & + \\
\hline Etomé & & + & Marsassoum & + & \\
\hline Kamobeul & & + & Mangaroungou & + & \\
\hline Mandjak & & + & Diana Malari & + & \\
\hline Emaye & & + & Diatakounda & & + \\
\hline Dioère & & + & Balmadou & + & \\
\hline Oukout & & + & Diafar & + & \\
\hline BIGNONA & + & + & Diafar Santo & & + \\
\hline Boulataye & + & & Madina & & + \\
\hline \multirow[t]{2}{*}{ Diatang } & & + & Guindir Bôlon & & + \\
\hline & & & Djibabouya & & + \\
\hline
\end{tabular}


Tableau récapitulatif des prospections sur les glossines (suite)

\begin{tabular}{|c|c|c|c|c|c|}
\hline Localités & $\begin{array}{l}\text { o. morsitans } \\
\text { submorsitans }\end{array}$ & $\begin{array}{r}\text { G. palpaits } \\
\text { gambiensis }\end{array}$ & Localitës & $\begin{array}{l}\text { G. morsitans } \\
\text { submorsitans }\end{array}$ & $\begin{array}{l}\text { G. palpalis } \\
\text { gambiensis }\end{array}$ \\
\hline DIOULOULOU & & + & Patiana & + & \\
\hline Sêlếty & & + & Wassadou & & + \\
\hline Birkamandine & & + & KOULOUNTOU & + & \\
\hline Narangs & + & & NIOKOLO KOBA & + & + \\
\hline KOULIMBA & & + & Salemata & & + \\
\hline Guiro & + & & Dienoundialo & + & \\
\hline Kandiadiou & + & & Mansandala & + & \\
\hline Diakoye & + & & Tiankoye & + & \\
\hline Sitoukène & + & & KEDQUGOU & + & + \\
\hline Balandine & & + & Mako & + & + \\
\hline KOLDA & + & + & Fongolimbi & + & \\
\hline Saré Sirilo & + & & Bandafassi & + & \\
\hline Temkilam Ilo & + & + & Binbou & + & \\
\hline Moltaba & + & & Saraya & + & \\
\hline Linguéto & + & & Missira & + & \\
\hline Saré NDiaye & & + & TAMBACOUNDA & + & \\
\hline Sarê Keita & & + & Mae1i Dibi & + & \\
\hline Faramba & & + & Maka & + & + \\
\hline Saré Diata & & + & Kolibantan & + & \\
\hline Mahon & + & + & Pakēba & + & + \\
\hline Bantangueul & & + & Koussanar-sud & + & \\
\hline Tiêval Lao & + & & Sandoumana & & + \\
\hline Sarê Abdoulaye & + & & BALA & + & \\
\hline FAFAKOUROU & + & & GOUDIRY & + & \\
\hline Badion & + & & Kotiari & + & \\
\hline Dinguiraye & + & & BAKEL & & \\
\hline Saré Maounda & + & & Sinthiou Tapsirou & + & \\
\hline Sarế D. So & + & & M. Samba Guiro & + & \\
\hline VELINGARA & + & & Toulēkêdi & + & \\
\hline KOUNKANE & + & & Boudel, Ndia & + & \\
\hline Kayanga & + & + & & & \\
\hline
\end{tabular}




\section{CARTE DE L'AFRIQUE DE L'OUEST}

FEUILLE ND 28-ПI

$1 / 200000$

Glossina palpalis gambiensıs

$\square$ Glossina morsitans submorsitans

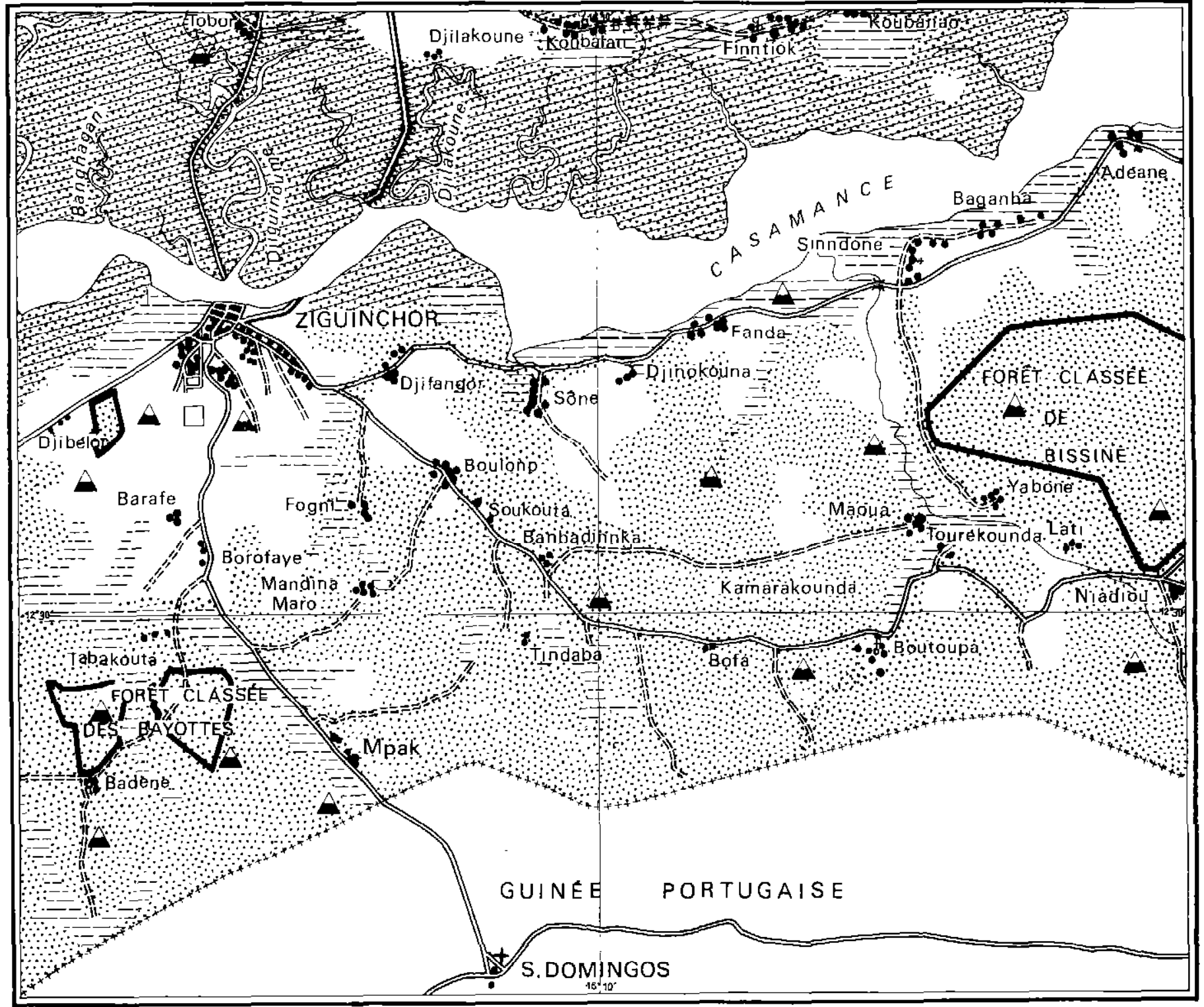




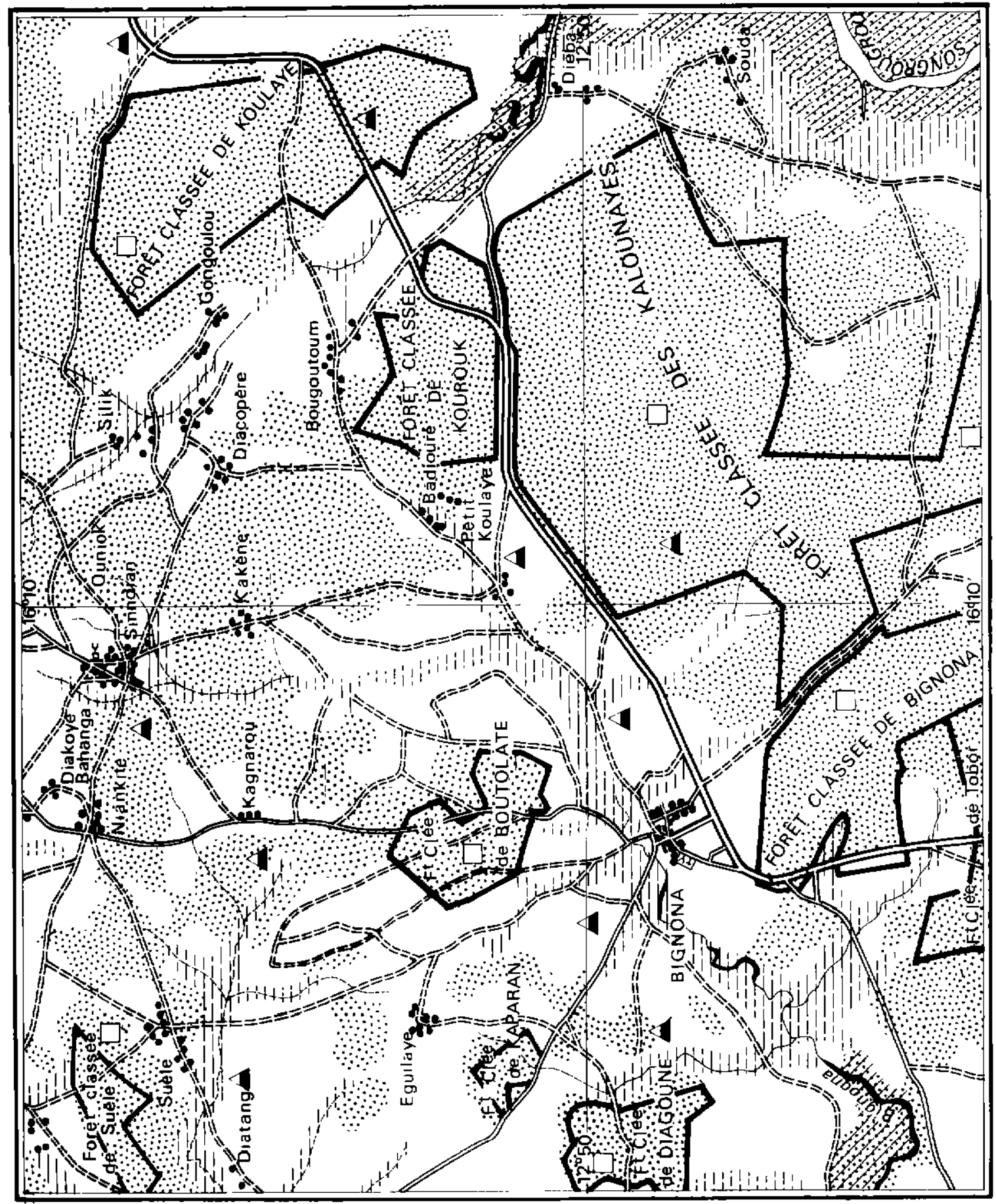

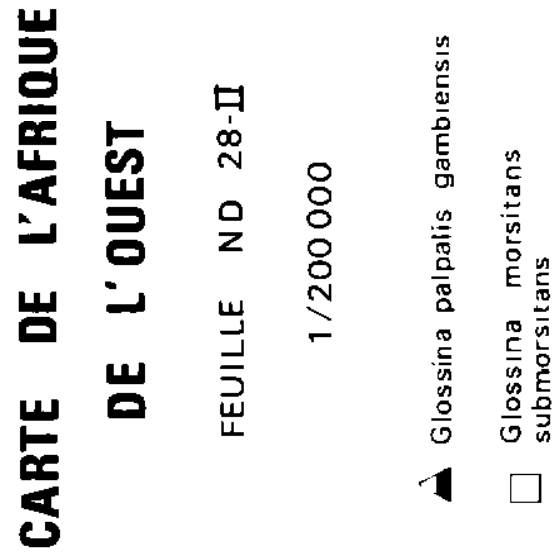




\section{CARTE DU SÉNEGAL}

\section{$1 / 500.000$}

Glossina morsitans submorsitans.

A Glossina palpalis gambiensis

Facies botanıques dominants : savane arborée et forêts claires

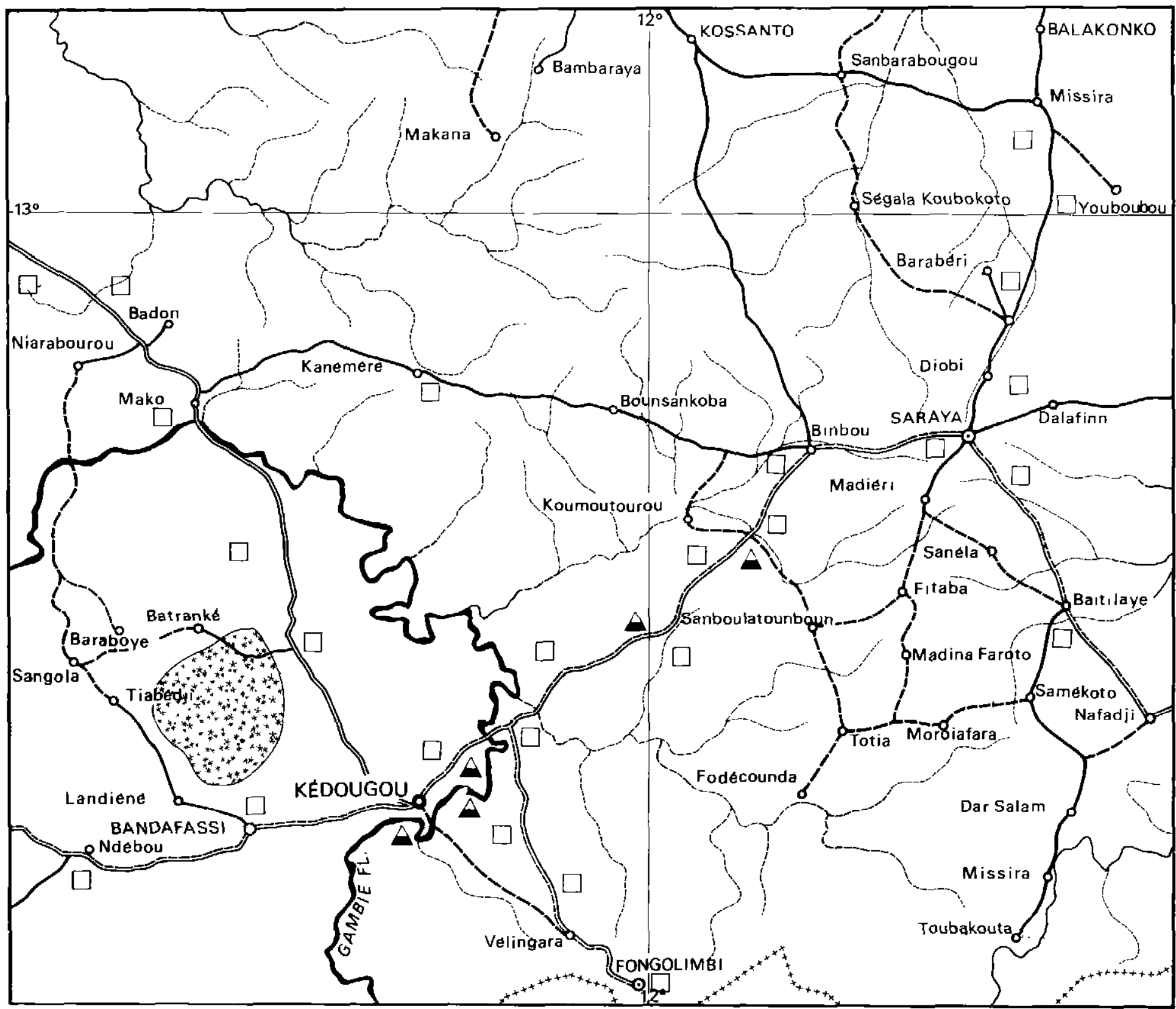


CARTE DU SENEgaL

$1 / 500000$

Glossina morsıtans submorsitans

Glossina palpalis gambiensıs

Zones prospectees sans glos sines.d'in festation peu probable ou d'infestation perioque et transitore

Facies botaniques dominants savane au nord.

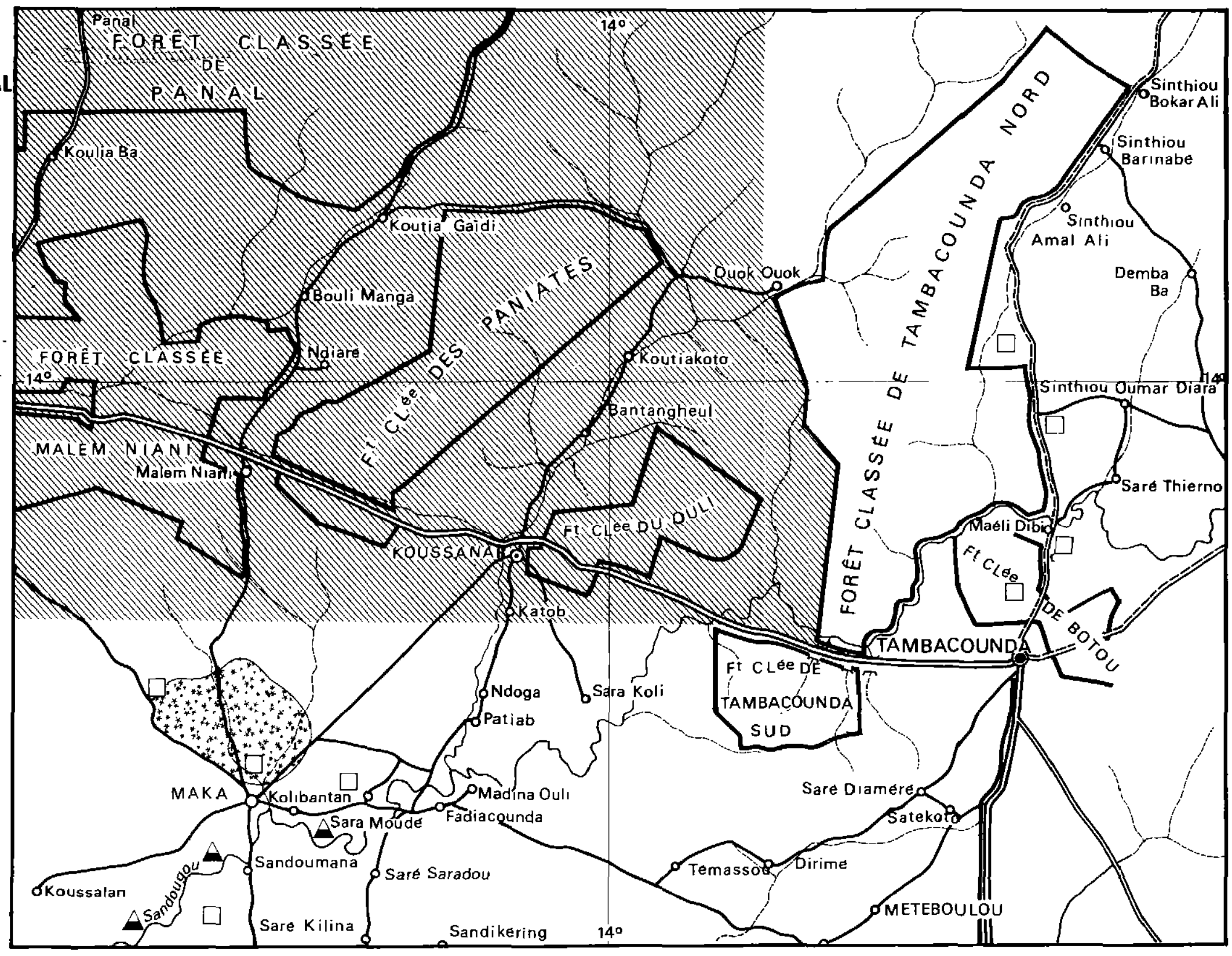




\section{SUMMARY}

\section{Glossina (Diptera, Glossinidae) in Senegal : Ecology, geographical distribution and their incidence on trypanosomiasis}

This is a synthesis of the data from entomological survey for Glossina species which took place in Senegal from 1965 to 1968 . Three Glossina species are to be mentioned: G. morsitans submorsitans Newstead, 1910; G. longipalpis Wiedemann, 1830; $G$. palpalis gambiensis Vanderplank, 1949.

Glossina species occupy the southern part of Senegal, except some located areas along the atlantic coasts where $G$. palpalis gambiensis is still to be found in palm-groves of guinean type and in dense vegetation along some small rivers.

In the mangrove swamps of the Saloum river-mouth $G$. palpalis gambiensis is quite frequent whereas $G$. morsitans submorsitans occupies the woodland.

The most important Glossina populations were found in Casamance and in Eastern Senegal. In these countries $G$. palpalis gambiensis is present in several types of vegetation: moist forest, palm-groves, mangrove swamps and waterside bordering wood; $G$. morsitans submorsitans has larger distribution areas, mainly the woodlands and the savannah woodlands.

$G$. longipalpis had been reported in a previous study but was not caught again during these present fly-rounds.

On the area of Glossina distribution domestic animals harbour one or several Trypanosoma species: $T$. brucei, $T$. congolense, $T$. vivax. Bovine trypanosomiasis is fairly tolerated in Casamance and in Upper Gambia where the breeding cattle is the Ndama type but in other countries of Zebu cattle animals suffer from the disease.

To the present is joined a general distribution map.

\section{RESUMEN}

Las glosinas (Diprera, Glossinidae) de Senegal : ecologia, repartición geográfica e incidencia sobre las tripanosomiasis

Esta nota presenta una sintesis de las investigaciones hechas entre 1965 y 1968 para precisar la repartición geográfica y la ecologia de las glosinas en Senegal.

Se mencionan tres especies de glosinas: Glossina morsitans submorsitans Newstead, 1910; G. longspalpis Wiedemann, 1830; G. palpalis gambiensis Vanderplank, 1949.

Las glosinas ocupan el sur de Senegal, salvo algunos islotes de $G$. palpalis gambiensis situados a lo largo de la costa atlántica en palmerales o marismas con vegetación densa.

En el manglar de la embocadura del Saloum, G. palpalis gambiensis es frecuente, mientras que se encuentra $G$. morsitans en las selvas claras.

Se encuentra el más gran número de glosinas en Casamance y Senegal oriental. En dichas regiones, $G$. palpalis está en varios tipos de formaciones botanicas : las selvas densas húmedas, las palmerales, el manglar y la vegetación ribereña de los ríos. $G$. morsitans submorsitans tiene una vasta repartición en las selvas claras y la sabana arborescente. No se descubrió durante estas inyestigaciones $G$. longipalpis, mencionada por trabajos anteriores.

En la área ocupada por las glosinas, los animales domésticos albergan una o varias especies de tripanosomos: Trypanosoma brucei, T. congolense, $T$. vivax, pero la tripanosomiasis se tolera relativamente bien en Casamance y en Alta Gambia (donde el ganado es de raza Ndama) en relación con otras regiones de ganaderia de cebues.

Una mapa general de repartición de las glosinas ilustra este estudio.

\section{BIBLIOGRAPHIE}

1. BOUET (G.), Contribution à l'étude des zones à glosşines dú Sénégal (région du chemin de fer, de Thiès à Kayes). Bull. Soc. Path. exot., 1916: 9, 802-13.

2. BUXTON (P.A.), The natural history of the tse-tse flies. (An account of the biology of the genus Glossina (Diptera), London Sch. Hyg-trop. Med., 1955, 10, 816 p.

3. CHALLIER (A.), EYRAUD (M.) et Collab., Ecologie de Glossina palpalis gambiensis Vander- 
Retour au menu

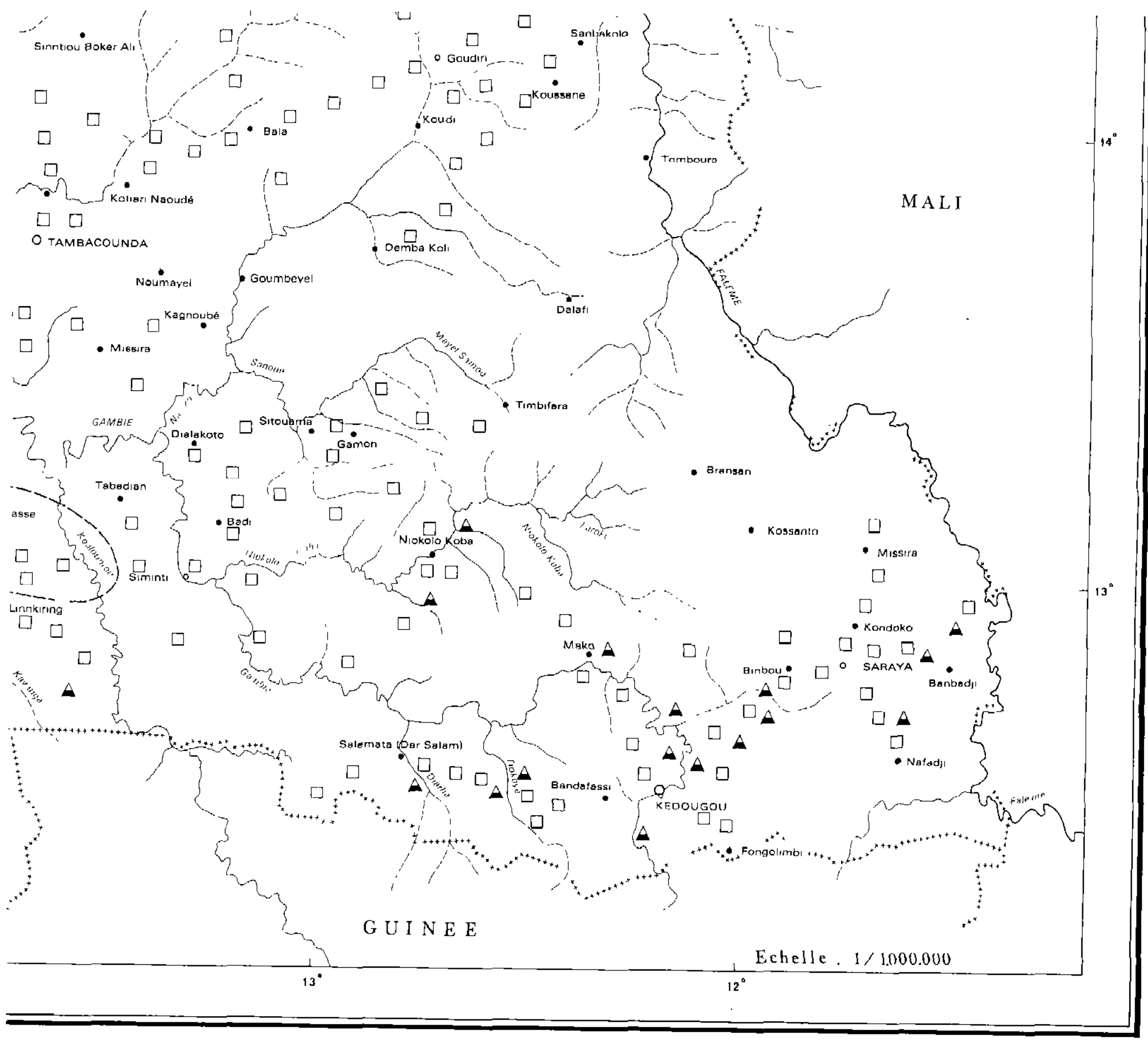

A Glossura paipails gambuensis

Institul a Elevage et de Médecine vetermaire

dor Pays Tropicaux - Massons-Alion - Val de Marne

D Glossına morsitang submorsitans 


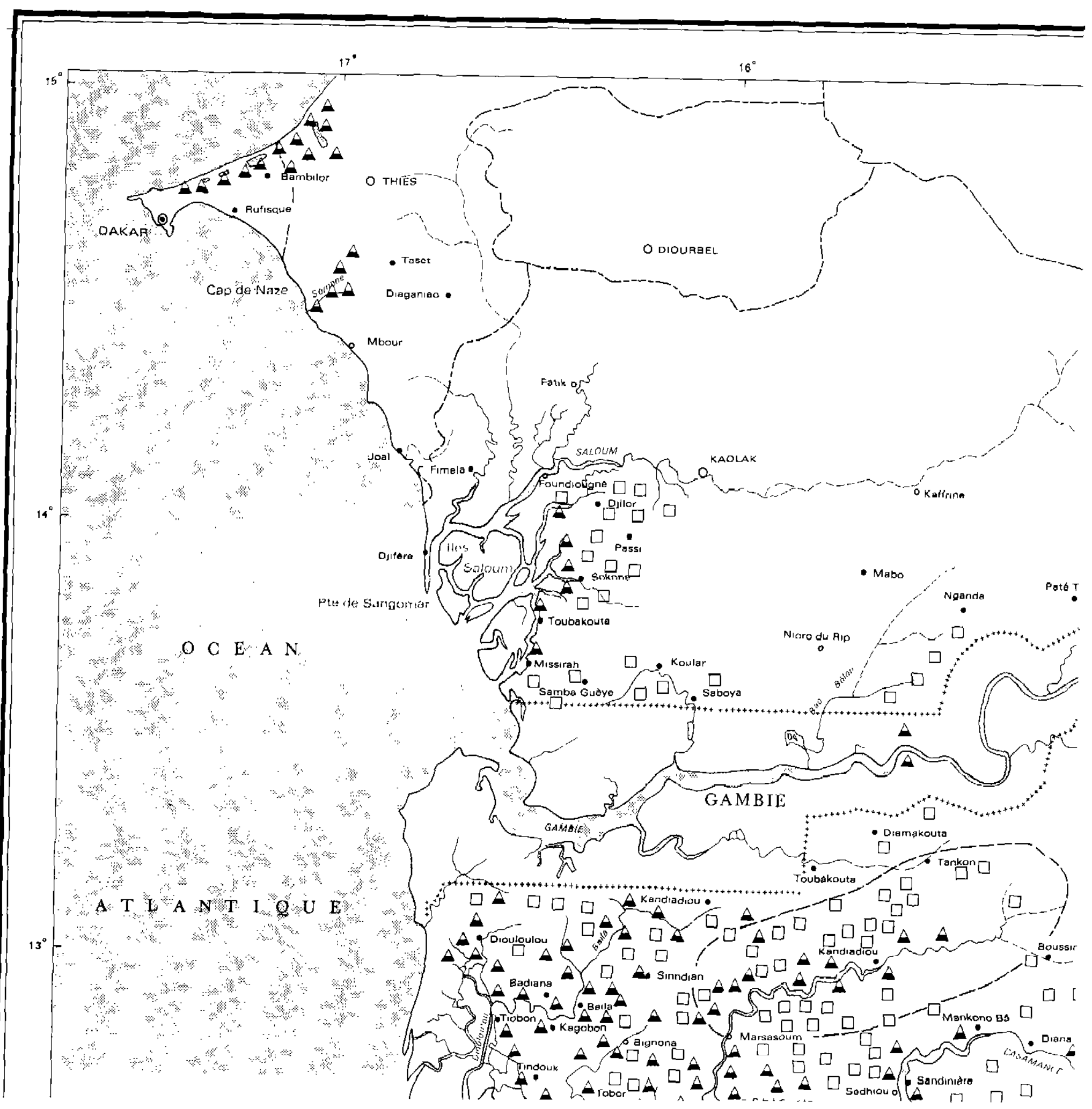


plank, 1949 et épidémiologie de la trypanosomiase humaine dans le foyer de la Petite-Côte, République du Sénégal. Enquête du 25 mars au 25 avril 1970, Rapport polycopié, Bobo-Dioulasso, Centre Muraz, no 119/ENT/70.

4. DUFOUGERE (M. W.), La maladie du sommeil et les trypanosomiases animales en Casamance, J. off. Afr. occident. fr., 1912 (69) (supplementum), 7 p.

5. FINELLE (P.), ITARD (J.), YVORE (P.), LACOTTE (R.), Répartition des glossines en République Centrafricaine. Etat actuel des connaissances, Rev. Elev. Méd. vét. Pays trop., 1963, 16 (3) : $337-48,4$ cartes.

6. FORD (J.), The distribution of the vectors of african pathogenic trypanosomes, Bull. Org. Mond. Santé, 1963, 28 (5-6) : 653-69.

7. GASCHEN (H.), Les glossines de l'Afrique occidentale française, Acta trop., 1945, supplementum 2, $131 \mathrm{p}$.

8. GOUZIEN (P.), Répartition topographique de la maladie du sommeil dans l'Afrique tropicale française, Publ. Association Scient. intern. Agronom. colon., Paris, Levé, 1908, pp. 26-31.

9. KEAY (R.W. J.), Vegetation map of Africa South of the tropic of Cancer, London, Oxford University Press, 1959, 24 p.

10. MACHADO (A. de BARROS), Révision systématique des glossines du groupe palpalis (Diptera), Publ. Serv. cultur, Companhia Diamanses Angola, Lisboa, 1954, 22, 189 p.

11. MOREL (P. C.), TOURE (S. M.), Glossina palpalis gambiensis Vanderplank, 1949 (Diptera) dans la région des Niayes et sur la Petite-Côte (Rép. du Sénégal). Rev. Elev. Méd. vét. Pays trop., 1967, 20 (4) : 571-78.

12. MORNET (P.), Carte de répartition des trypanosomes pathogènes des animaux domestiques en Afrique occidentale française, Bull. Soc. Path. exot., 1953, 41 (3) : 308-11.

13. MOULANIER (M.), DIOUF (J.), Dix années de lutte contre la maladie du sommesl sur la Petite-
Côte du Sénégal et la région des Niayes, Bull. Méd. Afr. occident. fr., 1952, 9 (2) : 201-38.

14. NEWTEAD (R.), Guide to the study of the tse-tse flies, Liverpool Sch, trop. Med., 1924, 1 (n.s.), 332 p.

15. POTTS (W.H.), Distribution of tse-tse species in Africa, London, Directorate of Colonial Surveys, 1953, 3 cards colour. sheet 1 : West Africa.

16. RICKENBACH (A.), Carte de répartition des glossines en Afrique occidcntale d'cxpression française, Paris, O.R.S.T.O.M., 1961.

17. ROUBAUD (E.), Les mouches tsé-tsé dans l'Ouest africain. Distribution géographique. Histoire - Rôle pathogène - I. La distribution géographique des tsé-tsé, Ann. Inst. Pasteur, Paris, $1915,36(10): 720-28,1$ carte h.t.

18. Services géographiques de l'A.O.F., Limites des aires de distribution de Glossina palpalis et Glossina tachinoides, Glossina morsitans et $G$. longipalpis en Afrique occidentale, 2 cartes, 1949.

19. THIROUX (A.), Les villages de ségrégation de traitement de la maladie du sommeil. Fonctionnement d'un de ces villages à Saint-Louis du Sénégal, J. off. Afr. occident. fr., 1909 (7), supplementum, 8 p.

20. THIROUX (A.), ANFREVILLE DE LA SALLE (L. de), La maladie du sommeil et les trypanosomiases animales au Sénégal, Paris, Baillières, $1909,200 \mathrm{p}$.

21. TOURE (S. M.), Glossines et trypanosomiases au Sénégal. Rapports sur les enquêtes effectuées entre 1965 et 1968, Dakar, Laboratoire national de l'Elevage et de Recherches vétérinaires.

22. TOURE (S. M.), Répartition géographique et écologie spéciale des glossines (Diptera, Muscidae) au Sénégal, in: Control of livestock insect pests by the sterile male technique, Vienna, International Atomic Energy Agency, 1968: 89-93.

23. TOURE (S. M.), XXVI - Diptera Glossinidae in : Le Parc national du Niokolo-Koba (Sénégal). Fascicule III, Mém. Institut fondamental d'Afrique noire, 1969 (84) : 397-400. 